\title{
COMMUTATIVITY IN OPERATOR ALGEBRAS
}

\author{
DAVID P. BLECHER
}

(Communicated by Paul S. Muhly)

\begin{abstract}
By an 'operator algebra' we shall mean a subalgebra $\mathscr{A}$ of the algebra $\mathscr{B}(\mathscr{H})$ of bounded operators on a Hilbert space $\mathscr{H}$, together with the matrix normed structure $\mathscr{A}$ inherits from $\mathscr{B}(\mathscr{H})$. A unital operator algebra is an operator algebra with an identity of norm 1 . Note that we do not require the algebra to be self-adjoint or uniformly closed. Such algebras were characterized abstractly by Ruan, Sinclair, and the author up to complete isometric isomorphism. In this paper we study commutativity for operator algebras, and we give a characterization of commutative unital operator algebras and a characterization of unital uniform algebras.
\end{abstract}

By an 'operator algebra' we shall mean a subalgebra $\mathscr{A}$ of the algebra $\mathscr{B}(\mathscr{H})$ of bounded operators on a Hilbert space $\mathscr{H}$, together with the matrix normed structure $\mathscr{A}$ inherits from $\mathscr{B}(\mathscr{H})$. A unital operator algebra is an operator algebra with an identity of norm 1 . Note that we do not require the algebra to be self-adjoint or uniformly closed. Such algebras were characterized abstractly in [1] up to complete isometric isomorphism. In this paper we study commutativity for operator algebras, and we give a characterization of commutative unital operator algebras and a characterization of unital uniform algebras.

We begin with some definitions (see also $[6,15,18]$ ). If $\mathscr{H}$ is a Hilbert space, and if $\mathscr{B}(\mathscr{H})$ is the algebra of bounded operators on $\mathscr{H}$, then the algebra $\mathscr{M}_{n}(\mathscr{B}(\mathscr{H}))$ of $n \times n$ matrices with entries in $\mathscr{B}(\mathscr{H})$ has a natural norm $\|\cdot\|_{n}$, namely via the identification $\mathscr{M}_{n}(\mathscr{B}(\mathscr{H}))$ with $\mathscr{B}\left(\mathscr{H}^{(n)}\right)$, where $\mathscr{H}^{(n)}$ is the direct sum of $n$ copies of $\mathscr{H}$. A linear subspace $X$ of $\mathscr{B}(\mathscr{H})$ inherits this natural norm on the space $\mathscr{M}_{n}(X)$; we call $X$, or more precisely the pair $\left(X,\|\cdot\|_{n}\right)$, an operator space. If $T: X \rightarrow Y$ is a linear map between operator spaces then $T$ induces a natural map $T_{n}: \mathscr{M}_{n}(X) \rightarrow \mathscr{M}_{n}(Y)$ given by $T_{n}\left(\left[x_{i j}\right]\right)=\left[T\left(x_{i j}\right)\right]$; we say that $T$ is completely bounded if

$$
\|T\|_{c b}=\sup \left\{\left\|T_{n}\right\|: n \in \mathbf{N}\right\}<\infty .
$$

Also $T$ is said to be a complete contraction if $\|T\|_{c b} \leq 1$, and a complete isometry if each $T_{n}$ is an isometry.

Received by the editors April 3, 1989 and, in revised form, July 18, 1989; presented at S.E.A.M. V, Athens, Georgia, March 31-April 1, 1989.

1980 Mathematics Subject Classification (1985 Revision). Primary 46K05, 47D25. 
If $\left(X,\|\cdot\|_{n}\right)$ is an operator space then we define $X^{\text {op }}$, the opposite operator space to $X$, to be the operator space $\left(X,\|\cdot\|_{n}^{\mathrm{op}}\right)$ where

$$
\left\|\left[x_{i j}\right]\right\|_{n}^{\mathrm{op}}=\left\|\left[x_{j i}\right]\right\|_{n}
$$

for $\left[x_{i j}\right] \in \mathscr{M}_{n}(X)$. If $\mathscr{A}$ is a $C^{*}$-algebra then $\mathscr{A}^{\text {op }}$ may be identified with the $C^{*}$-algebra which has the same Banach space structure as $\mathscr{A}$, but with the reversed multiplication. If $X$ is an operator space contained in a $C^{*}$ algebra $\mathscr{A}$ then $X^{\text {op }}$ may be identified as a subspace of the $C^{*}$-algebra $\mathscr{A}^{\text {op }}$. We define $\operatorname{SYM}(X)$, the symmetrized space of $X$, to be the operator space $\left(X, \max \left\{\|\cdot\|_{n},\|\cdot\|_{n}^{\text {op }}\right\}\right)$. If $X$ is contained in a $C^{*}$-algebra $\mathscr{A}$ then this operator space is readily identified as the subspace

$$
\left\{(x, x) \in \mathscr{A} \oplus \mathscr{A}^{\mathrm{op}}: x \in X\right\}
$$

of $\mathscr{A} \oplus \mathscr{A}^{\text {op }}$ (where $\oplus$ is the direct sum of $C^{*}$-algebras). We say that an operator space $X$ is symmetric if the identity mapping $X \rightarrow \operatorname{SYM}(X)$ is a complete isometry; or in other words if $\|\cdot\|_{n}=\|\cdot\|_{n}^{\text {op }}$ for each $n$.

T. Okayusu showed in [13] that a $C^{*}$-algebra is symmetric if and only if it is a commutative algebra. J. Tomiyama proved a related result in [21]. Notice that not every commutative unital operator algebra is symmetric. To see this let $X$ be any operator space which is not symmetric, and suppose $X \subseteq \mathscr{B}(\mathscr{H})$. The subalgebra of $\mathscr{M}_{2}(\mathscr{B}(\mathscr{H}))$ consisting of elements of the form

$$
\left[\begin{array}{ll}
\lambda & x \\
0 & \lambda
\end{array}\right]
$$

for $\lambda \in \mathbf{C}$ and $x \in X$, is unital and commutative, but not symmetric.

Proposition 1. If $\mathscr{A}$ is an operator algebra then $\mathscr{A}^{\mathrm{op}}$ is an operator algebra with the reversed multiplication.

Proof. This is simply the fact that if $\mathscr{A}$ is a subalgebra of a $C^{*}$-algebra $\mathscr{B}$ then $\mathscr{A}^{\text {op }}$ with the reversed multiplication is a subalgebra of the $C^{*}$-algebra $\mathscr{B}^{\text {op }}$.

We shall also need the following fact.

Proposition 2. A surjective unital complete isometry of unital operator algebras is an isomorphism.

Proof. Let $\mathscr{A}$ and $\mathscr{B}$ be unital operator algebras and suppose $\Psi: \mathscr{A} \rightarrow \mathscr{B}$ is a unital complete isometry. Suppose $\mathscr{A} \subseteq \mathscr{B}(\mathscr{H})$; we may assume without loss of generality that the identity operator $I_{\mathscr{P}}$ is the identity of $\mathscr{A}$. Define a new pseudo-multiplication $m$ on $\mathscr{A}$ by $m\left(a_{1}, a_{2}\right)=\Psi^{-1}\left(\Psi\left(a_{1}\right) \Psi\left(a_{2}\right)\right)$ for $a_{1}, a_{2} \in \mathscr{A}$. Now $m$ is completely contractive as a bilinear map, and has identity $I_{\mathscr{H}}$. [1, Corollary 2.4] shows that both $m$ and the usual multiplication on $\mathscr{A}$ are the restriction to $\mathscr{A}$ of the multiplication on the injective envelope of $\mathscr{A}$ (which is a $C^{*}$-algebra). 
Remark. We note that the above proposition also appears in [7] with a different proof, and was discovered independently. Subsequently V. I. Paulsen has pointed out to the author that the result follows readily from [9, 4.1 and 4.2] and considering the $C^{*}$-envelopes of $\mathscr{A}+\mathscr{A}^{*}$ and $\mathscr{B}+\mathscr{B}^{*}$. The proposition may be viewed as the noncommutative generalization of Nagasawa's result for uniform algebras: a unital isometry of unital uniform algebras is a homomorphism [12]. Indeed the last statement follows immediately from the proposition and [15, Theorem 3.9].

Okayusu used Kadison's result on isometries between $C^{*}$-algebras [11] to prove that symmetry implies commutativity for $C^{*}$-algebras. The same reasoning, using Proposition 2, gives the result for unital operator algebras:

Theorem 3. Let $\mathscr{A}$ be a unital operator algebra. The following are equivalent:

(i) $\mathscr{A}$ is a commutative algebra,

(ii) $\mathscr{A}$ with the reversed multiplication is an operator algebra,

(iii) $\mathscr{A}^{\text {op }}$ is an operator algebra with the usual multiplication of $\mathscr{A}$,

(iv) $\operatorname{SYM}(\mathscr{A})$ is an operator algebra with the usual multiplication of $\mathscr{A}$,

(v) $\operatorname{SYM}(\mathscr{A})$ is an operator algebra with the reversed multiplication.

Remark. We reiterate our convention that an 'operator algebra' is a subalgebra of some $B(\mathscr{H})$ together with the accompanying matrix norms. Thus, for example, condition (ii) above means that the underlying operator space of $\mathscr{A}$, considered together with the reversed multiplication, is completely isometrically isomorphic to a subalgebra of some $\mathscr{B}(\mathscr{H})$; or equivalently [1], that the reversed multiplication is completely contractive.

Proof of Theorem 3. That (ii) is equivalent to (iii) and that (iv) is equivalent to (v) follows from Proposition 1. Clearly (i) implies (ii), and Proposition 2 shows that (ii) implies (i). Since (ii) implies (i) it follows that either of the equivalent conditions (iv) or (v) will imply the commutativity of $\operatorname{SYM}(\mathscr{A})$ (from which (i) follows). Thus it is only necessary to show that (iii) implies (iv). However since $\operatorname{SYM}(\mathscr{A})$ is completely isometrically imbedded in the direct sum $\mathscr{A} \oplus_{\infty} \mathscr{A}^{\text {op }}$ via the map $a \rightarrow a \oplus a$ the desired conclusion follows.

We are now able to characterize the unital symmetric operator algebras.

Corollary 4. If $\mathscr{A}$ is a unital symmetric operator algebra then $\mathscr{A}$ is a commutative algebra. Conversely, if $\mathscr{A}$ is a commutative operator algebra then $\operatorname{SYM}(\mathscr{A})$ is a commutative symmetric operator algebra.

Remark. The results above are probably true for nonunital algebras, but we are unable to give a proof.

Recall that a uniform algebra is a uniformly closed subalgebra of the algebra of continuous functions on a compact Hausdorff space. In fact we shall not require that the algebra be uniformly closed. Now a uniform algebra $\mathscr{A}$ has a natural operator space structure, inherited from the containing commutative 
$C^{*}$-algebra. When considering a uniform algebra as an operator space it shall always be with reference to this particular structure.

More generally, if $X$ is any normed linear space then there is a canonical 'smallest' operator space structure on $X$. This is the one obtained by considering $X$ as a subspace of $C\left(\operatorname{BALL}\left(X^{*}\right)\right)$, the space of continuous functions on the compact Hausdorff space which is the unit ball of the dual space of $X$ with the weak* topology. We denote this operator space by $\operatorname{MIN}(X)$. Equivalently, one may specify the matrix norms by the identification

$$
\mathscr{M}_{n}(\operatorname{MIN}(X))=\mathscr{M}_{n} \otimes_{\lambda} X,
$$

where $\lambda$ is the injective tensor norm (see [6]). If $\mathscr{A}$ is a uniform algebra considered as an operator space as in the preceding paragraph then $\mathscr{A}=\operatorname{MIN}(\mathscr{A})$, of course.

We shall call an operator space which is of the form $\operatorname{MIN}(X)$, for some normed space $X$, a commutative operator space. The following result shows that the word 'commutative' here is less ambiguous than it might seem at first sight.

Theorem 5. Let $\mathscr{A}$ be a unital operator algebra which is a commutative operator space. Then $\mathscr{A}$ is completely isometrically isomorphic to a uniform algebra.

Proof. It is easy to see from Corollary 4 that $\mathscr{A}$ is a commutative algebra; however, we shall require a different approach to show that $\mathscr{A}$ is actually a uniform algebra.

Suppose $\mathscr{B}$ is the injective envelope of $\mathscr{A}$ [19]. It is shown in [1, Corollary $2.4]$ that $\mathscr{B}$ is a $C^{*}$-algebra containing $\mathscr{A}$ as a subalgebra. Now $\mathscr{A}$ considered as a normed space possesses an injective envelope $E[4,10]$, and it is not hard to see that $\operatorname{MIN}(E)$ is an injective envelope for the operator space $\mathscr{A}$. By the uniqueness of injective envelopes $\mathscr{B}=\mathrm{MIN}(E)$ completely isometrically, so that $\mathscr{B}$ is a commutative operator space. Thus by Corollary 4 (or indeed by Okayusu's result, or by [20, Theorem 4.14]) it follows that $\mathscr{B}$ is a commutative $C^{*}$-algebra. Thus $\mathscr{B}$ is of the form $C(\Omega)$ for some compact Hausdorff space $\Omega$; since $\mathscr{A}$ is a subalgebra of $\mathscr{B}$ we see that $\mathscr{A}$ is a uniform algebra.

Remark. The unital assumption is critical here. For if $X$ is a commutative operator space contained in $\mathscr{B}(\mathscr{H})$ say, and if $\mathscr{A}$ is the algebra of elements of $\mathscr{M}_{2}(\mathscr{B}(\mathscr{H}))$ of the form

$$
\left[\begin{array}{ll}
0 & x \\
0 & 0
\end{array}\right],
$$

where $x \in X$, then $\mathscr{A}$ is a commutative operator space and an operator algebra, but obviously not a uniform algebra.

In [8] Grothendieck defined a tensor norm $H$ on the algebraic tensor product $X \otimes Y$ of two normed spaces $X$ and $Y$. This norm, corresponding to factorization through a Hilbert space, is also known as $\gamma_{2}$ [17]; and it may be 
defined as follows:

$$
\|u\|_{H}=\inf \left\{\sup \left\{\left(\sum_{i=1}^{n}\left|f\left(x_{i}\right)\right|^{2}\right)^{1 / 2}\left(\sum_{i=1}^{n}\left|g\left(y_{i}\right)\right|^{2}\right)^{1 / 2}\right\}\right\}
$$

for $u \in X \otimes Y$, where the supremum is taken over all $f \in \operatorname{BALL}\left(X^{*}\right)$ and all $g \in \operatorname{BALL}\left(Y^{*}\right)$, and the infimum is taken over all representations $u=$ $\sum_{i=1}^{n} x_{i} \otimes y_{i}$ of $u$.

The Haagerup operator space tensor norm $\|\cdot\|_{h}$ was introduced in $[5,16]$. Now if $X$ and $Y$ are any two normed spaces we may regard them as commutative operator spaces in the way outlined above, and form the Haagerup tensor product $\operatorname{MIN}(X) \otimes_{h} \operatorname{MIN}(Y)$. This is again an operator space [16], but will not in general be a commutative operator space. Now consider $\operatorname{MIN}(X) \otimes_{h} \operatorname{MIN}(Y)$ regarded as a normed vector space. This is the (uncompleted) tensor product of the normed spaces $X$ and $Y$ with respect to a certain normed space tensor norm which we now identify.

Proposition 6. If $X$ and $Y$ are normed spaces then $\operatorname{MIN}(X) \otimes_{h} \operatorname{MIN}(Y)$ is linearly isometrically isomorphic to $X \otimes_{H} Y$.

Proof. If $X$ is a normed space then $\operatorname{MIN}(X)$ has the operator space structure inherited from the usual inclusion of $X$ in the $C^{*}$-algebra of continuous functions on the compact set $\Omega=\operatorname{BALL}\left(X^{*}\right)$, the unit ball of the dual of $X$ in the weak* topology. Now there is a canonical correspondence between $M_{n, m}(C(\Omega))$ and $C\left(\Omega ; M_{n, m}\right)$; and if $x=\left[x_{1}, x_{2}, \ldots, x_{n}\right]$ is a matrix in $M_{1, n}(\mathrm{MIN}(X))$ then the norm of $x$ is precisely its norm as an element of $C\left(\Omega ; M_{1, n}\right)$. It follows that

$$
\begin{aligned}
\|x\| & =\sup \left\{\left\|\left[x_{1}(\omega), \ldots, x_{n}(\omega)\right]\right\|: \omega \in \Omega\right\} \\
& =\sup \left\{\left\|\left[f\left(x_{1}\right), \ldots, f\left(x_{n}\right)\right]\right\|: f \in \operatorname{BALL}\left(X^{*}\right)\right\} \\
& =\sup \left\{\left(\sum\left|f\left(x_{i}\right)\right|^{2}\right)^{1 / 2}: f \in \operatorname{BALL}\left(X^{*}\right)\right\} .
\end{aligned}
$$

Exactly the same identity holds if instead $x=\left[x_{1}, x_{2}, \ldots, x_{n}\right]^{t}$ is a matrix in $M_{n, 1}(\operatorname{MIN}(X))$. Thus if $X$ and $Y$ are normed spaces, and

$$
u \in M_{1}(\operatorname{MIN}(X) \otimes \operatorname{MIN}(Y))
$$

then

$$
\begin{aligned}
\|u\|_{h} & =\inf \left\{\|x\|\|y\|: x \in M_{1, n}(\operatorname{MIN}(X)), y \in M_{n, 1}(\operatorname{MIN}(Y)), u=x \odot y\right\} \\
& =\|u\|_{H} .
\end{aligned}
$$

Remark. Once it is seen that the Haagerup norm $\|\cdot\|_{h}$ is the noncommutative generalization of Grothendieck's $H$-norm many results in the theory of the Haagerup norm and completely bounded multilinear maps may be seen to be noncommutative generalizations of known results in the metric theory of tensor products of Banach spaces. For a discussion of this see [2, §4]. 
We recall that if $\mathscr{A}$ is a normed algebra and if $\alpha$ is the tensor norm defined on $\mathscr{A} \otimes \mathscr{A}$ then $\mathscr{A}$ is called an $\alpha$-algebra if the canonical map $\mathscr{A} \otimes A \rightarrow \mathscr{A}$ given by $a \otimes b \rightarrow a b$ is a contraction when $\mathscr{A} \otimes \mathscr{A}$ is equipped with the $\alpha$ norm. A. M. Tonge [22] characterized unital uniform algebras up to isometric isomorphism by showing that they were precisely the $d_{2}$-algebras (where $d_{2}$ is a tensor norm which we shall not define here).

An easy consequence of the main theorem in [1] is the following characterization of unital uniform algebras.

Theorem 7. A unital normed algebra $\mathscr{A}$ is isometrically isomorphic to a uniform algebra if and only if it is an $\mathrm{H}$-algebra.

Proof. The necessity is obvious; indeed any uniform algebra is a $\lambda$-algebra. Conversely if $\mathscr{A}$ is a unital $H$-algebra then by Proposition 6 the canonical map $\operatorname{MIN}(\mathscr{A}) \otimes_{h} \operatorname{MIN}(\mathscr{A}) \rightarrow \operatorname{MIN}(\mathscr{A})$ is contractive, and consequently completely contractive by [15, Theorem 3.9]. Now it follows from [1] that $\operatorname{MIN}(\mathscr{A})$ is an operator algebra, and Theorem 5 shows that $\mathscr{A}$ is a uniform algebra.

Remark 1. It is not necessary to assume that $\mathscr{A}$ is associative in Theorem 7. However the example following Theorem 5 and [1, Example 4.1] shows that the unital condition cannot be removed.

Remark 2. Theorem 7 is the commutative analogue of the characterization of uniform algebras given in [1]. Indeed the main result of [1] may be rephrased as follows: a unital $L^{\infty}$-Banach algebra is an operator algebra if and only if it is a 'matricial' $\|\cdot\|_{h}$-algebra. Carne showed in [3] that the class of operator algebras cannot be described by a single Banach space tensor norm; this does not contradict the above. Parrott's example $[14,15]$ of a uniform algebra with a contractive homomorphism which is not completely contractive shows that we cannot expect a completely isometric isomorphism in Theorem 7.

Remark 3. Since Grothendieck's $H$ tensor norm is dominated by the $d_{2}$ norm it is clear that Theorem 7 follows from Tonge's result, at least in the case when $\mathscr{A}$ is associative. However the proof given here is conceptually very simple, and besides treats the nonassociative case.

\section{ACKNOWLEDGMENT}

The author wishes to thank V. I. Paulsen for many helpful comments and conversations. Acknowledgment is also due to the seminal papers [23].

\section{REFERENCES}

1. D. P. Blecher, Z-J. Ruan and A. M. Sinclair, A characterization of operator algebras, J. Funct. Anal. (to appear).

2. D. P. Blecher and V. I. Paulsen, Tensor products of operator spaces, preprint.

3. T. K. Carne, Not all $H^{\prime}$-algebras are operator algebras, Math. Proc. Cambridge Philos. Soc. 86 (1979), 243-249. 
4. H. B. Cohen, Injective envelopes of Banach spaces, Bull. Amer. Math. Soc. 70 (1964), $723-$ 726.

5. E. G. Effros, On multilinear completely bounded maps, Contemp.Math. 62 (1987), 450-479.

6. E. G. Effros and Z-J. Ruan, On matricially normed spaces, Pacific J. Math. 132 (1988), 243-264.

7. __ On non-self-adjoint operator algebras, preprint.

8. A. Grothendieck, Resume de la theorie metrique des produits tensoriels topologiques, Bull. Soc. Mat. Sao Paulo 8 (1956), 1-79.

9. M. Hamana, Injective envelopes of operator systems, Publ. RIMS Kyoto Univ. 15 (1979), 773-785.

10. J. R. Isbell, Three remarks on injective envelopes of Banach spaces, J. Math. Anal. 27 (1969), 516-518.

11. R. V. Kadison, Isometries of operator algebras, Ann. of Math. 54 (1951), 325-338.

12. M. Nagasawa, Isomorphisms between commutative Banach algebras with applications to rings of analytic functions, Kōdai Math. Sem Rep. 11 (1959), 182-188.

13. T. Okayusu, Some cross norms which are not uniformly cross, Proc. Japan Acad. 46 (1970), 54-57.

14. S. K. Parrott, Unitary dilations for commuting contractions, Pacific J. Math. 34 (1970), 481-490.

15. V. I. Paulsen, Completely bounded maps and dilations, Pitman Research Notes in Math., Longman, London, 1986.

16. V. I. Paulsen and R. R. Smith, Multilinear maps and tensor norms on operator systems, J. Funct. Anal. 73 (1987), 258-276.

17. G. Pisier, Factorization of linear operators and geometry of Banach spaces, C.B.M.S. Series no. 60, Amer. Math. Soc., Providence, RI, 1986.

18. Z-J. Ruan, Subspaces of $C^{*}$-algebras, J. Funct. Anal. 76 (1988), 217-230.

19. _ Injectivity and operator spaces, Trans. Amer. Math. Soc. 315 (1989), 89-104.

20. M. Takesaki, Theory of operator algebras I, Springer-Verlag, Berlin, 1979.

21. J. Tomiyama, On the transpose map of matrix algebras, Proc. Amer. Math. Soc. 88 (1983), 635-638.

22. A. M. Tonge, Banach algebras and absolutely summing operators, Math. Proc. Cambridge Philos. Soc. 80 (1976), 465-473.

23. W. B. Arveson, Subalgebras of $C^{*}$-algebras I, Acta Math. 123 (1969), 142-224; II, 128 (1972), 271-308.

Department of Mathematics, University of Houston, Houston, Texas 77204-3476 